\title{
Influence of Relaxing Defects on the Paraelectric-Ferroelectric Phase Transitions of Rochelle Salt
}

\author{
J. Petersson \\ Fachbereich Physik der Universität des Saarlandes, D-6600 Saarbrücken
}

Z. Naturforsch. 34 a, 538-547 (1979); received February 24, 1979

\begin{abstract}
It is well known that different types of crystal defects influence the static and dynamic behaviour of the dielectric polarization of Rochelle salt crystals. Particularly strong effects appear near the paraelectric-ferroelectric phase transitions. In this paper it is shown that a slightly modified relaxing defect cell model which has been developed [1] in order to explain the narrow central peaks observed near structural phase transitions adequately describes the interaction between the strongly temperature dependent relaxational motion of the order parameter and the relaxational motions of the various defect modes. This theory is shown to be equivalent to a phenomenological Landau Khalatnikov approach, which so far has only been used to characterize the macroscopic dielectric behaviour of Rochelle salt crystals with defects near the phase transitions, and which in a previous work [16], has been developed in order to give a general description of central peak phenomena caused by defects. For instance the so called very fast after effect and its influence on the phase transitions is discussed in more detail.
\end{abstract}

\section{Introduction}

It has been shown by experiments which have been performed on a variety of materials that near structural phase transitions very narrow central peaks often appear in addition to the soft phonon peaks. The widths and the intensities of the central peaks depend strongly on the temperature near the phase transition. In a recent work Halperin and Varma (HV) [1] have pointed out that the behaviour of the central peak may be understood by taking into account the dynamics of certain defects. In the relaxing defect cell model a defect in the unit cell is considered which may hop back and forth between two equilibrium positions and which couples linearly to the order parameter. The characteristic time scale of this motion determines the width of the central peak. Until now, however, there is only little direct experimental evidence for the role of defects in central peak dynamics. In a recent neutron scattering study of the structural phase transition in $\mathrm{SrTiO}_{3}$ it has been shown [2], that the intensity of the central peak of hydrogen reduced $\mathrm{SrTiO}_{3}$ is systematically enhanced with increasing defect concentration. The transition temperature decreases linearly with increasing defect concentration. These results clearly show that defects are involved in the central peak mechanism of $\mathrm{SrTiO}_{3}$. They also demonstrate, however, that the relaxing defect cell model cannot

Reprint requests to Prof. Dr. J. Petersson. Please order a reprint rather than making your own copy.

$0340-4811 / 79 / 0500-0538 \$ 01.00 / 0$ account for the observed behaviour since it predicts an increasing $T_{c}$ with increasing defect concentration. Rather the "frozen" impurity model [1] eventually may explain this central peak type.

Rochelle salt - a double tartrate of sodium and potassium of composition $\mathrm{NaKC}_{4} \mathrm{H}_{4} \mathrm{O}_{6} \cdot 4 \mathrm{H}_{2} \mathrm{O}-$ was the first crystal to be identified as a ferroelectric. The crystal undergoes phase transitions of the second order at about 24 and $-19^{\circ} \mathrm{C}$. In the upper and lower phases the crystal is paraelectric whereas between these temperatures the ferroelectric phase appears. The sole ferroelectric axis is parallel to the orthorhombic $a$-axis and the crystal is of the piezoelectric type, i.e. even in the paraelectric phase there is a linear coupling between the critical dielectric and the elastic behaviour. It has been well established $[3,4,5]$ that an orderdisorder mechanism drives the phase transition. As a consequence a strong monodispersive relaxational dispersion of the dielectric constant can be detected in the microwave regime. Both parameters of this dispersion - the relaxation strength and the relaxation time - depend strongly on the temperature in similar manners. On the other hand, it has been shown by many works [6-14] that several types of defects characteristically influence the frequency and temperature behaviour of the macroscopic dielectric properties of Rochelle salt. It is the main purpose of the present work to show that the well known interaction between these relaxing defect modes and the critical relaxing dielectric mode follows exactly the formalism of the HV relaxing defect cell model. 
One important fact should be stressed. As has been mentioned earlier, the critical dynamics of the order parameter is of the relaxational type. This behaviour, which has been detected in many other order-disorder ferroelectrics, would result in a strongly temperature dependent central peak, which may be regarded as an intrinsic property of an undisturbed crystal without any defects. Obviously this central peak plays a role at orderdisorder transitions which is similar to the roles of the peaks of the soft phonons at displacive structural phase transitions. It is not only this central peak which will be discussed in the following. Rather we are mainly interested in its interaction with the relaxing defect modes in the crystal.

So far the influence of the crystal defects on the dynamical dielectric behaviour of Rochelle salt has only been taken into account by applying the formalism of the thermodynamics of irreversible processes [14-16]. In these works it is assumed that, besides the relaxational macroscopic order parameter, there exists another relaxational thermodynamic variable of state which may be attributed to the defects and which couples linearly to the order parameter. It will be shown below that this way of describing the influence of the defects is equivalent to that introduced in the $\mathrm{HV}$ model. In particular it has been shown by the author of the present work [16] that - within the frame of these theories - a linear coupling of a relaxing defect mode and an oscillatory soft mode gives rise to a strongly temperature dependent central peak near a displacive structural phase transition. Theories of this type may be regarded as extended Landau Khalatnikov [17] theories.

In the following section the HV formalism will be rewritten to the special situation of Rochelle salt. In section III a Landau Khalatnikov (LK) approach is presented and its equivalence is established with the HV formalism. Finally in section IV the influence of defects on the dielectric dynamics of Rochelle salt will be discussed in more detail, with emphasis on some special cases and the underlying physical mechanisms.

\section{Halperin and Varma Formalism}

In order to rewrite the $\mathrm{HV}$ formalism to the special situation of Rochelle salt the following assumptions are introduced. (i) The dynamical behaviour of the order parameter of a perfect crystal is of the relaxational type $[3,4,5]$. This implies that for the wave vector $q=0$ the quantity $\left(-\omega^{2} m+i \omega \gamma+x_{n}\right)$ of the $\mathrm{HV}$ model is to be replaced by $\left(i \omega \tau_{n}+1\right) x_{n}$, where $\tau_{n}$ is the relaxation time of the "normal" ferroelectric order parameter in the non-interacting system.

(ii) A further relaxational variable is introduced which describes the dynamical behaviour of the defect modes. It is assumed that the perfect lattice cells contain the ferroelectric dipoles whereas the disturbed lattice cells contain both the ferroelectric and the defect dipoles. Obviously this fact does not hold for the original $\mathrm{HV}$ model in which it is assumed that a lattice cell may be either a perfect cell or a defect cell (the following results may be easily rewritten to the $\mathrm{HV}$ model). As a consequence the quantity $(1-c)$ of the $\mathrm{HV}$ model is to be replaced by 1 .

(iii) Since Rochelle salt is a piezoelectric ferroelectric it is important to fix the elastic boundary conditions in a definite manner. It will be assumed that in the entire temperature and frequency range the condition of a free crystal is realized. This condition generally cannot be realized experimentally. In the range of the characteristic frequency of the order parameter the crystal is clamped. On the other hand the condition of a free crystal can be realized in the range of the characteristic frequency of the defects. However, principally the size of the sample may be assumed to be so small that the piezoelectric resonances appear at such high frequencies that the condition of a free crystal is realized in the entire frequency range of interest. It is important to note that one of the defect relaxations in Rochelle salt is strongly influenced by the elastic boundary conditions. This effect will be discussed in Section IV.

It should be noted that in the piezoelectric ferroelectrics the linear coupling between the polarization fluctuations and acoustical phonons gives rise to a central peak in the Brillouin scattering spectrum. Effects of this type have been detected e.g. in Rochelle salt [5] and crystals of the KDP group [18-20]. They are described by a coupled mode approach $[5,18]$ which in some sense corresponds to the LK theory to be presented in the next section. Thus, from a formal point of view the linear coupling between the critical ferroelectric mode on the one hand and the accoustic 
phonons or the defect modes on the other hand may be handled in similar manners. In the following we will not be concerned explicitely with the piezoelectric coupling between the ferroelectric mode and the elastic degrees of freedom.

(iv) In order to simplify the discussion the nonpolar phases will be discussed only. The results may be easily extended to the polar phase by taking into account the arguments presented in Refs. $[1,14,15]$. Furthermore we will restrict the discussion to the wave vector $\boldsymbol{q}=0$. This is because in ferroelectric crystals the instability appears for modes in the centre of the Brillouin zone and there exist well developed experimental methods which enable to measure the complex dynamical dielectric susceptibility near $\boldsymbol{q}=\mathbf{0}$.

On these conditions the frequency dependent susceptibility matrix $\chi(\omega)$ of the HV model is given by

$\chi^{-1}(\omega)=\left(\begin{array}{ccc}\left(i \omega \tau_{n}+1\right) x_{n}-J & -c J \\ -J & \left(i \omega \tau_{d}+1\right) x_{d}-c J\end{array}\right)$.

The symbols in this relation have the usual meaning. The $\chi(\omega)$ matrix may be written as

$$
\chi(\omega)=\left(\begin{array}{ll}
\chi_{n n}(\omega) & \chi_{n d}(\omega) \\
\chi_{d n}(\omega) & \chi_{d d}(\omega)
\end{array}\right)
$$

In an investigation of the dielectric behaviour generally the mean susceptibility $\bar{\chi}(\omega)$ is measured. With respect to assumption (ii) the following relation is assumed to hold

$$
\bar{\chi}(\omega)=\chi_{n n}+\chi_{n d}+c\left(\chi_{d n}+\chi_{d d}\right) .
$$

In dielectric measurements near ferroelectric phase transitions this quantity plays a role similar to that of the form factor in quasi elastic scattering experiments at structural phase transitions. Writing the determinant of the $\chi(\omega)$ matrix in the form

$$
\begin{aligned}
\Delta(\omega)= & \Delta_{s}+i \omega\left(\tau_{n} x_{n}\left(x_{d}-c J\right)\right. \\
& \left.+\tau_{d} x_{d}\left(x_{n}-J\right)\right) \\
& -\omega^{2} \tau_{n} x_{n} \tau_{d} x_{d}
\end{aligned}
$$

where

$$
\Delta(0)=\Delta_{s}=x_{n} x_{d}-J\left(c x_{n}+x_{d}\right)
$$

denotes the static value of $\Delta(\omega)$ the mean susceptibility may be written as

$$
\bar{\chi}(\omega)=\left(x_{d}+c x_{n}+i \omega\left(\tau_{d} x_{d}+c \tau_{n} x_{n}\right)\right) \Delta^{-1}(\omega) .
$$

The static value $\bar{\chi}_{s}$ of $\bar{\chi}(\omega)$ is given by

$$
\bar{\chi}(\omega=0)=\bar{\chi}_{s}=\left(x_{d}+c x_{n}\right) \Delta_{s}^{-1} .
$$

The dynamical susceptibility of the undisturbed crystal, i.e. $c=0$, may be written as

$$
\bar{\chi}^{0}(\omega)=\bar{\chi}_{s}{ }^{0}\left(1+i \omega \tau_{\varepsilon}^{0}\right)^{-1},
$$

where

$$
\bar{\chi}_{s}^{0}=x_{d}\left(\Delta_{s}^{0}\right)^{-1}=\left(x_{n}-J\right)^{-1},
$$

and

$$
\tau_{\varepsilon}^{0}=\tau_{n} x_{n} \bar{\chi}_{s}^{0}
$$

are the static susceptibility and the relaxation time of the order parameter, respectively. Assuming the mean field approximation which for most of the ferroelectrics constitutes a good approximation, we may write

$$
\left(\bar{\chi}_{s}{ }^{0}\right)^{-1}=\left(T-T_{c}{ }^{0}\right) / C,
$$

where $C$ is the Curie constant and $T_{c}{ }^{0}$ is the limit of stability of the non ordered phase. At $T=T_{c}{ }^{0}$ the relation $x_{n}=J$ holds. In accordance with the $\mathrm{HV}$ model all critical temperature-dependences will be deduced from Equation (11). Since $\tau_{n} x_{n}$ generally depends in a non-critical manner on the temperature according to a thermally activated process near $T_{c}{ }^{0}$ the relaxation time $\tau_{\varepsilon}^{0}$ behaves similarly to $\bar{\chi}_{s}{ }^{0}$ (critical slowing down). Equations (8)-(10) represent the dynamical susceptibility for many order-disorder ferroelectrics in particular for Rochelle salt [3-5]. TGS [21] and $\mathrm{AgNa}\left(\mathrm{NO}_{2}\right)_{2}$ [22] constitute examples of special interest.

Turning now to a crystal with defects, i.e. $c>0$, according to Eqs. (5), (7) and (9) the static susceptibility is given by

$$
\bar{\chi}_{s}^{-1}=\left(\bar{\chi}_{s}\right)^{-1}-c x_{n}^{2}\left(x_{d}+c x_{n}\right)^{-1} .
$$

Thus, in accordance with the HV model a CurieWeiß law

$$
\bar{\chi}_{s}^{-1}=\left(T-T_{c}\right) / C
$$

is obtained with an unchanged Curie constant $C$ and a higher Curie temperature $T_{c}$ which is given by

$$
T_{c}=T_{c}^{0}+C c x_{n}^{2}\left(x_{d}+c x_{n}\right)^{-1} .
$$

Obviously in first approximation this shift is proportional to the defect concentration $c$ (Figure 1). At the phase transition temperature $T_{c}$ the relation

$$
x_{n}^{-1}+c x_{d}^{-1}=J^{-1}
$$

holds.

Discussing now the dynamical behaviour of a crystal with defects Eq. (6) may be written for a complex $z$ instead of $i \omega$ in the form

$$
\bar{\chi}(z)=\bar{\chi}_{s} f(z),
$$




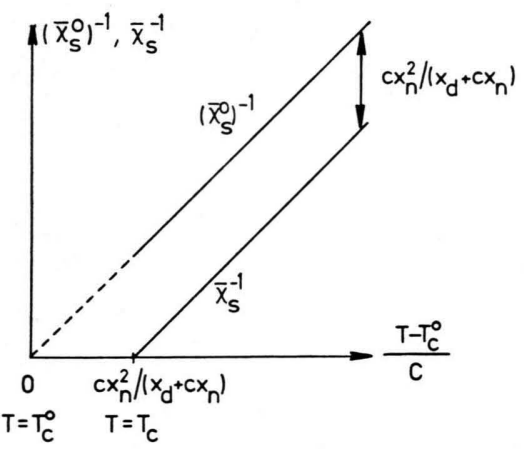

Fig. 1. Schematic representation of the temperature dependences of the static susceptibilities $\bar{\chi}_{\mathrm{s}}{ }^{0}$ of a perfect crystal and $\bar{\chi}_{\mathrm{s}}$ of a crystal with defects.

where $f(z)$ is given by

$$
f(z)=(1+\alpha z)\left(1+\beta z+\gamma z^{2}\right)^{-1}
$$

and $\alpha, \beta$ and $\gamma$ are positive constants which can be expressed by the quantities appearing in Eqs. (4) and (6). It is important to note that both quantities $\beta$ and $\gamma$ are proportional to $\bar{\chi}_{s}$ whereas $\alpha$ exhibits no critical behaviour. The zero's of the denominator of $f(z)$ are

$$
z_{1,2}=-\left(1 \mp\left(1-4 \gamma / \beta^{2}\right)^{1 / 2}\right) \beta / 2 \gamma
$$

and thus

$$
\begin{aligned}
f(z)= & z_{2}\left(1+\alpha z_{1}\right)\left(z_{2}-z_{1}\right)^{-1}\left(1-z / z_{1}\right)^{-1} \\
& +z_{1}\left(1+\alpha z_{2}\right)\left(z_{1}-z_{2}\right)^{-1}\left(1-z / z_{2}\right)^{-1} .
\end{aligned}
$$

An elementary but lengthy calculation which will not be presented here gives the following exact results. The numerators $z_{2}\left(1+\alpha z_{1}\right)\left(z_{2}-z_{1}\right)^{-1}$ and $z_{1}\left(1+\alpha z_{2}\right)\left(z_{1}-z_{2}\right)^{-1}$ which appear in Eq. (19) are both real, not negative and smaller than or equal to one. Both zero's $z_{1}$ and $z_{2}$ are real and negative. Consequently for $z=i \omega$ the complex susceptibility can be represented by

$\bar{\chi}(\omega)=\bar{\chi}_{s 1}\left(1+i \omega \tau_{\varepsilon 1}\right)^{-1}+\bar{\chi}_{s 2}\left(1+i \omega \tau_{\varepsilon 2}\right)^{-1}$.

Obviously $\bar{\chi}(\omega)$ is a linear superposition of two independent susceptibilities which can be attributed to relaxational motions. The corresponding relaxation times and static susceptibilities $\bar{\chi}_{s 1}$ and $\bar{\chi}_{s 2}$ are defined by

$$
\begin{aligned}
\tau_{\varepsilon 1}^{-1} & =-z_{1}>0, \quad \tau_{\varepsilon 2}^{-1}=-z_{2}>0 . \\
0 \leqq \bar{\chi}_{s 1} & =\bar{\chi}_{s} z_{2}\left(1+\alpha z_{1}\right)\left(z_{2}-z_{1}\right)^{-1} \leqq \bar{\chi}_{s}, \\
0 \leqq \bar{\chi}_{s 2} & =\bar{\chi}_{s} z_{1}\left(1+\alpha z_{2}\right)\left(z_{1}-z_{2}\right)^{-1} \leqq \bar{\chi}_{s},
\end{aligned}
$$

respectively. Note that $\bar{\chi}_{s}=\bar{\chi}_{s 1}+\bar{\chi}_{s 2}$.
The temperature dependences of $z_{1}$ and $z_{2}$ determine those of the relaxation times and of the static susceptibilities. It will be shown that the temperature behaviour of the dynamic susceptibility in Eq. (20) corresponds to a central peak type behaviour. Arranging the relaxation frequencies according to

$$
0 \leqq \tau_{\varepsilon 2}^{-1} \leqq \tau_{\varepsilon 1}^{-1}
$$

and taking into account Eqs. (10), (18), and (21) at the phase transition $T=T_{c}$

$\tau_{\varepsilon 2}^{-1}=0, \quad \tau_{\varepsilon 1}^{-1}=\left(\tau_{\varepsilon}{ }^{0}\right)^{-1}+\left(x_{d}-c J\right)\left(\tau_{d} x_{d}\right)^{-1}$.

Thus, on approaching $T_{c}$ the relaxation frequency $\tau_{\varepsilon 2}^{-1}$ tends to zero whereas $\tau_{\varepsilon 1}^{-1}$ approaches a value which is larger than the relaxation frequency of the order parameter of the undisturbed crystal at the same temperature. According to Eq. (18) the relation $\tau_{\varepsilon 1} \tau_{\varepsilon 2}=\gamma$ holds which implies that $\tau_{\varepsilon 1} \tau_{\varepsilon 2}$ follows a Curie-Weiß law. In particular the product

$$
\tau_{\varepsilon 1} \tau_{\varepsilon 2} \bar{\chi}_{s}^{-1}=\tau_{n} x_{n} \tau_{d} x_{d}\left(x_{d}+c x_{n}\right)^{-1}
$$

shows no critical temperature dependence. The static susceptibilities and their behaviours for $T \rightarrow T_{c}$ can be represented by

$$
\begin{aligned}
& \bar{\chi}_{s 1} / \bar{\chi}_{s}=\left(\alpha-\tau_{\varepsilon 1}\right) /\left(\tau_{\varepsilon 2}-\tau_{\varepsilon 1}\right) \rightarrow 0, \\
& \bar{\chi}_{s 2} / \bar{\chi}_{s}=\left(\tau_{\varepsilon 2}-\alpha\right) /\left(\tau_{\varepsilon 2}-\tau_{\varepsilon 1}\right) \rightarrow 1 .
\end{aligned}
$$

Thus, on approaching $T_{c}$ both the relaxation time $\tau_{\varepsilon 2}$ and the corresponding relaxation strength $\bar{\chi}_{s 2}$ diverge. On the contrary, both the relaxation time

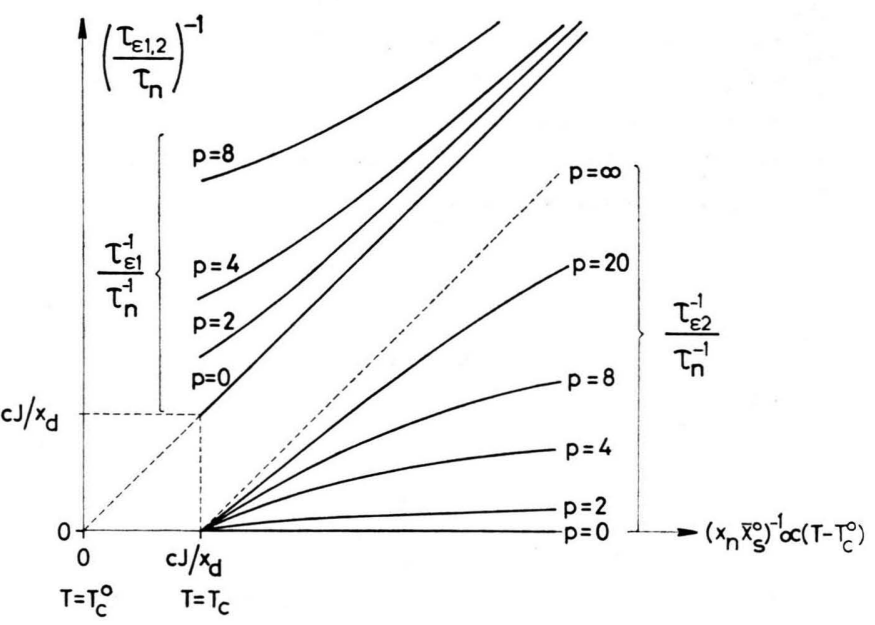

Fig. 2. Temperature dependence of the relaxation frequencies $\tau_{\varepsilon 1}^{-1}$ and $\tau_{\varepsilon 2}^{-1}$ as calculated from Equation (27). The parameter $p$ is assumed to be temperature independent. 
$\tau_{\varepsilon 1}$ and the corresponding relaxation strength $\bar{\chi}_{s 1}$ have finite values at $T_{c}$ (note that $\bar{\chi}_{s}$ obeys the Curie-Weiß law of Equation (13)).

Taking into account Eqs. (18), (21) and the meaning of the constants $\beta$ and $\gamma$ the approximate formula

$$
\begin{aligned}
& 2 \tau_{\varepsilon 1,2}^{-1} / \tau_{n}{ }^{-1}=\left(\left(x_{n} \bar{\chi}_{s}^{0}\right)^{-1}+p\right) \\
& \quad \pm\left[\left(\left(x_{n} \bar{\chi}_{s}\right)^{-1}+p\right)^{2}-4 p\left(x_{n} \bar{\chi}_{s}\right)^{-1}\right]^{1 / 2}
\end{aligned}
$$

can be deduced where the parameter

$$
p=\tau_{n} / \tau_{d}
$$

is the ratio of the characteristic time scales of the motions of the ferroelectric dipoles and the defects, respectively. Thus, with the aid of Eqs. (11) and (12) the temperature dependence of $\tau_{\varepsilon 1}$ and $\tau_{\varepsilon 2}$ can be derived (Figure 2).

\section{Landau Khalatnikov Approach}

The complex dielectric function $\varepsilon(\omega)$ may be written as

$$
\varepsilon(\omega)=\varepsilon_{\infty}+\bar{\chi}(\omega),
$$

where $\varepsilon_{\infty}$ is the real temperature independent dielectric constant at such high frequencies that $\bar{\chi}(\omega)$ vanishes. Then the following linear thermodynamic relations can be established

$$
\begin{aligned}
D & =\varepsilon_{\infty} E+P_{\varepsilon 1}+P_{\varepsilon 2}, \\
-l_{\varepsilon 1} \dot{P}_{\varepsilon 1}=-A_{\varepsilon 1} & =-E+\bar{\chi}_{s 1}^{-1} P_{\varepsilon 1}, \\
-l_{\varepsilon 2} \dot{P}_{\varepsilon 2}=-A_{\varepsilon 2} & =-E+\bar{\chi}_{\varepsilon 2}^{-1} P_{\varepsilon 2} .
\end{aligned}
$$

In these relations $E$ and $D$ denote the macroscopic electric field strength and the dielectric displacement, respectively. These quantities may be regarded as external thermodynamic variables of state. The macroscopic internal variables of state $P_{\varepsilon 1}$ and $P_{\varepsilon 2}$ have the meaning of independent normal dielectric polarizations. Their conjugates are the affinities $A_{\varepsilon 1}$ and $A_{\varepsilon 2}$ which vanish in equilibrium, i.e. $\dot{P}_{\varepsilon 1}=\dot{P}_{\varepsilon 2}=0$. The kinetic coefficients $l_{\varepsilon 1}$ and $l_{\varepsilon 2}$ are related to the quantities of the $\mathrm{HV}$ model by

$$
l_{\varepsilon 1}=\tau_{\varepsilon 1} \bar{\chi}_{s 1}^{-1}, \quad l_{\varepsilon 2}=\tau_{\varepsilon 2} \bar{\chi}_{s 2}^{-1} .
$$

Their temperature dependences can be derived from Eqs. (21), (22) and (25). It turns out that besides $\bar{\chi}_{s 1}$ and $\bar{\chi}_{s 2}$ also these quantities are affected by the phase transition. An order of magnitude calculation gives $l_{\varepsilon 1} \approx \tau_{n} x_{n}$ whereas $l_{\varepsilon 2}$ generally shows a more complicated behaviour. For a crystal without defects $P_{\varepsilon 2}=0$ and $P=P_{\varepsilon 1}$ is the order parameter of the phase transition. In this case according to Eq. (10) $l_{\varepsilon}=\tau_{n} x_{n}$.

The Eqs. (30)-(32) may be regarded as the fundamental relations of a thermodynamic theory $[15-17,23,24]$. They can be derived from an expansion of the corresponding thermodynamic potential in the variables $E, P_{\varepsilon 1}$ and $P_{\varepsilon 2}$ on the one hand and by taking into account the entropy production caused by the dynamical behaviour of $P_{\varepsilon 1}$ and $P_{\varepsilon 2}$ on the other hand. This formalism may be extended also to other cases of interest. If the soft mode does not appear in the centre of the Brillouin zone then $E$ and $D$ may be regarded as an external force and its conjugated displacement at the wave vector of the soft mode. If the modes are of the oscillatory type then besides the relaxational variables of state also odd variables of state must be taken into consideration [23, 24].

It is, however, well known $[15,24]$ that a transformation to other independent external variables of state may give a better insight in the physical meaning of the constitutive relations. This procedure corresponds to a Legendre transformation, i.e. the corresponding potential is obtained as a function of $D, P_{\varepsilon 1}$ and $P_{\varepsilon 2}$. Thus, rewriting Eqs. (30) to (32) in these new variables and transforming to normal polarization variables $P_{\beta 1}, P_{\beta 2}$, i.e. $P_{\beta 1}+P_{\beta 2}=P_{\varepsilon 1}+P_{\varepsilon 2}$ but $P_{\beta 1} \neq P_{\varepsilon 1}, P_{\varepsilon 2}$ and $P_{\beta 2} \neq P_{\varepsilon 1}, P_{\varepsilon 2}$ the result can be written as

$$
\begin{aligned}
E & =\beta_{\infty} D-\beta_{\infty} P_{\beta 1}-\beta_{\infty} P_{\beta 2}, \\
-l_{\beta 1} \dot{P}_{\beta 1} & =-A_{\beta 1}=-\beta_{\infty} D+R_{\beta 1} P_{\beta 1}, \\
-l_{\beta 2} \dot{P}_{\beta 2} & =-A_{\beta 2}=-\beta_{\infty} D+R_{\beta 2} P_{\beta 2} .
\end{aligned}
$$

In these relations we denote by $\beta_{\infty}=\varepsilon_{\infty}^{-1}$ the high frequency value of the dielectric modulus. The frequency dependent complex dielectric modulus $\beta(\omega)=(\varepsilon(\omega))^{-1}$ can be written as

$$
\begin{aligned}
\beta(\omega)=\beta_{\infty} & -\Delta \beta_{1}\left(1+i \omega \tau_{\beta 1}\right)^{-1} \\
& -\Delta \beta_{2}\left(1+i \omega \tau_{\beta 2}\right)^{-1},
\end{aligned}
$$

where the relaxation strengths and relaxation times are defined by $\Delta \beta_{1}=\beta_{\infty}^{2} R_{\beta 1}^{-1}, \Delta \beta_{2}=\beta_{\infty}^{2} R_{\beta 2}^{-1}$ and $\tau_{\beta 1}=l_{\beta 1} R_{\beta 1}^{-1}, \quad \tau_{\beta 2}=l_{\beta 2} R_{\beta 2}^{-1}$. According to Eqs. (20), (29), and (37) the relaxation times $\tau_{\varepsilon 1,2}$ and $\tau_{\beta 1,2}$ are given by the poles of the dielectric function $\varepsilon(z)$ and of the dielectric modulus $\beta(z)$, respectively. 
The temperature dependences of the material quantities appearing in Eqs. (34)-(37) may be estimated from the HV model as follows. Since - at least near $T_{c}-\varepsilon_{\infty} \ll \bar{\chi}_{s}$ holds, the static dielectric modulus can be represented by

$$
\begin{aligned}
\beta_{s 2} & =\beta_{\infty}-\Delta \beta_{1}-\Delta \beta_{2}=\varepsilon_{s 2}^{-1} \\
& =\left(\varepsilon_{\infty}+\bar{\chi}_{s}\right)^{-1} \approx \bar{\chi}_{s}^{-1} .
\end{aligned}
$$

Taking into consideration Eq. (12) the quantity $\beta_{s 1}=\beta_{\infty}-\Delta \beta_{1}$ can be related to the static susceptibility $\bar{\chi}_{s}{ }^{0}$ of the undisturbed crystal according to

$$
\beta_{s 1}=\beta_{\infty}-\Delta \beta_{1} \approx\left(\bar{\chi}_{s}^{0}\right)^{-1} .
$$

(Note, that generally $\beta_{s 1} \neq\left(\varepsilon_{\infty}+\bar{\chi}_{s 1}\right)^{-1}$.) On the other hand the relaxation strength $\Delta \beta_{2}$ may be attributed to an influence of the defects by the relation

$$
\Delta \beta_{2} \approx\left(\bar{\chi}_{s}^{0}\right)^{-1}-\bar{\chi}_{s}^{-1} .
$$

With the aid of Eq. (12) it follows that $\Delta \beta_{2}$ should not depend critically on the temperature (Fig. 1), but it should be proportional to the concentration $c$ of the defects.

We now turn to a discussion of the temperature dependence of the dynamical behaviour as given by Equations (34)-(37). The generalization of the well known LST relation $[15,24-26]$ to relaxational processes can be written as

$$
\tau_{\beta 1} \tau_{\beta 2} \beta_{\infty}=\tau_{\varepsilon 1} \tau_{\varepsilon 2} \beta_{s 2} .
$$

Thus, with the aid of Eqs. (25) and (38) it follows that the product $\tau_{\beta 1} \tau_{\beta 2}$ does not show any critical temperature dependence. It should be noted that among others [15, 24-26] Barker [28] emphasized the significance of the LST relation for a discussion of central peak type phenomena. Furthermore if the relaxation frequencies $\tau_{\varepsilon 1,2}^{-1}$ and $\tau_{\beta 1,2}^{-1}$ are arranged according to their magnitude the following relation holds $[15,27]$

$$
\begin{aligned}
0 \leqq \tau_{\varepsilon 2}^{-1} & \leqq \min \left(\tau_{\beta 1}^{-1}, \tau_{\beta 2}^{-1}\right) \leqq \tau_{\varepsilon 1}^{-1} \\
& \leqq \max \left(\tau_{\beta 1}^{-1}, \tau_{\beta 2}^{-1}\right)
\end{aligned}
$$

The relations (41) and (42) are exact, i.e. they can be derived from Eqs. (34)-(36) without introducing any further restricting assumption.

In order to simplify the discussion it will be assumed now that $\Delta \beta_{2} \ll \beta_{\infty}$. This condition is fullfilled at least near $T_{c}$ since in this region $\Delta \beta_{2}$ is of the order of magnitude of $\beta_{\infty}-\Delta \beta_{1}$ which in turn is small if compared to $\beta_{\infty}$. This condition means that the influence of the defects may be regarded to be small in a certain sense. On this condition starting from Eqs. (34)-(36) the relaxation frequencies $\tau_{\varepsilon 1}^{-1}$ and $\tau_{\varepsilon 2}^{-1}$ can be calculated as the eigen values of a suitably defined $2 \times 2$ matrix. The result may be compared directly to Eq. (27) and consequently it can be stated that

$$
\tau_{\beta 1}=\tau_{n} x_{n} \beta_{\infty}^{-1}, \quad \tau_{\beta 2}=\tau_{d} .
$$

Thus, the relaxation times $\tau_{\beta 1}$ and $\tau_{\beta 2}$ have a simple physical meaning. The relaxation time $\tau_{\beta 1}$ of the polarization $P_{\beta 1}$ of a crystal with defects is equal to the relaxation time $\tau_{\beta}=\tau_{\varepsilon}^{0}\left(\bar{\chi}_{s}^{0}\right)^{-1} \beta_{\infty}^{-1}$ of the polarization $P$ (order parameter of the phase transition) of the ferroelectric dipoles of a perfect crystal. The relaxation time $\tau_{\beta 2}$ is the relaxation time $\tau_{d}$ of the contribution $P_{\beta 2}$ of the defects to the polarization. In particular both quantities do not show a critical temperature dependence near $T_{c}$. Thus, one may indentify the variables $P_{\beta 1}$ and $P_{\beta 2}$ with polarizations which can be attributed to the ferroelectric dipoles (order parameter of a perfect crystal) and the defects. It should be noted that similar statements do not hold for the variables $P_{\varepsilon 1}$ and $P_{\varepsilon 2}$ because these quantities are suitably defined linear combinations of the variables $P_{\beta 1}$ and $P_{\beta 2}$. Throughout our discussion no restrictions have been introduced concerning the characteristic time scales of the motions of the ferroelectric dipoles and of the defects. In particular it may be admitted that the defects move faster than the ferroelectric dipoles, i.e. the parameter $p$ of Eq. (28) may be a large quantity. In all cases, according to relation (42) the minimum value which can be achieved by the relaxation frequency $\tau_{\varepsilon 1}^{-1}$ is given by the smallest value of the relaxation frequencies $\tau_{\beta 1}^{-1}$ and $\tau_{\beta 2}^{-1}$.

In the foregoing considerations it has been shown that starting from the $\mathrm{HV}$ model linear relations (34) - (36) can be established between the macroscopic variables of state. None of the material quantities appearing in these relations shows a critical temperature dependence. Rather on approaching $T_{c}$ the static moduli $\beta_{s 1}, \beta_{s 2}$ and the relaxation frequencies $\tau_{\varepsilon 1}^{-1}$ and $\tau_{\varepsilon 2}^{-1}$ behave in an anomaleous manner. On the other hand in an LK approach [16] exactly this behaviour can ba derived from a basic assumption. From an expansion of the appropriate thermodynamic potential the linear relations $(34)-(36)$ can be derived. In this approach one may assume that none of the 
coefficients of these constitutive relations shows a critical temperature dependence. Taking into consideration the conditions of thermodynamic stability $[15,16]$, however, the following facts can be stated. For a perfect crystal on approaching $T_{c}{ }^{0}$ the determinant $\beta_{\infty} R_{\beta 1}-\beta_{\infty}^{2} \rightarrow 0$. This implies that $\beta_{s 1}$ and $\tau_{\varepsilon}{ }^{0}$ are proportional to $T-T_{c}{ }^{0}$, i.e. they follow Curie-Weiß laws (Eqs. (39), (10)). For a crystal with defects on approaching $T_{c}$ the determinant $\beta_{\infty} R_{\beta 1} R_{\beta 2}-\beta_{\infty}^{2} R_{\beta 1}-\beta_{\infty}^{2} R_{\beta 2} \rightarrow 0$. This implies that $\beta_{s 2} \propto T-T_{c}$ follows a shifted CurieWeiß law (Equation (38)). The difference

$$
\beta_{s 1}-\beta_{s 2}=\Delta \beta_{2}=\beta_{\infty}^{2} R_{\beta 2}^{-1}
$$

does not depend on the temperature (Equation (40)). Thus, for $\beta_{s 1}$ and $\beta_{s 2}$ Fig. 1 holds in the same way. Similarly $\tau_{\beta 1}, \tau_{\beta 2}$ may be regarded as some phenomenological time constants which define the time scales of the variables $P_{\beta 1}$ and $P_{\beta 2}$ and which both do not show any critical temperature dependence. The relaxation frequencies $\tau_{\varepsilon 1}^{-1}$ and $\tau_{\varepsilon 2}^{-1}$ can be calculated as the eigen values of a suitably defined $2 \times 2$ matrix, which becomes positive semidefinite at $T_{c}$. Also in this representation the LST relation (41) shows that the product $\tau_{\varepsilon 1} \tau_{\varepsilon 2}$ follows the same temperature dependence as $\beta_{82}^{-1}$. The temperature dependences of these quantities can be represented in a form similar to Fig. 2 (see Fig. 1 of Ref. [16]) by rewriting the quantities of the HV model to the corresponding macroscopic quantities of the LK model according to

$$
\begin{aligned}
\left(\bar{\chi}_{s}^{0}\right)^{-1} & \rightarrow \beta_{s 1} x_{n} \beta_{\infty}^{-1}, \\
\tau_{n} & \rightarrow \tau_{\beta 1}=\tau_{n} x_{n} \beta_{\infty}^{-1}, \\
p & \rightarrow p^{\prime}=p x_{n} \beta_{\infty}^{-1}=\tau_{\beta 1} / \tau_{\beta 2}, \\
c J x_{d} & \rightarrow c J x_{d} x_{n} \beta_{\infty}^{-1}=\Delta \beta_{2} \beta_{\infty}^{-1} .
\end{aligned}
$$

\section{Dielectric After Effects in Rochelle Salt}

Several after effects which interact with the Curie-Weiß law of the dielectric constant have been detected in Rochelle salt single crystals [6-14]. Generally these after effects give rise to a frequency dependence of the dielectric constant and to special effects in the ferroelectric phase, e.g. a frequency dependence of the coercive electric field intensity. In most cases the after effects in Rochelle salt crystals can be characterized by monodispersive relaxations. So far three after effects have been detected which follow the LK theory or the equivalent $\mathrm{HV}$ model. At room temperature the time constants (in the above notation $\tau_{\beta 2}$ ) of these most extensively studied dielectric relaxations caused by crystal defects in Rochelle salt are of the order of magnitude $100 \mathrm{~s}, 0.5 \mathrm{~ms}$, and $0.2 \mu \mathrm{s}$ (very fast after effect). The corresponding activation energies are $1.4,0.8$, and $0.5 \mathrm{eV}$ per particle, respectively. For the present purpose it suffices to discuss one of the observed after effects more in detail. We therefore restrict ourselves to a discussion of the so called very fast after effect $[7,8,28]$ paying special attention to a verification of the $\mathrm{HV}$ model. In addition some special properties of this after effect will be discussed.

The results of Fig. 3 demonstrate that in the lower paraelectric phase near the ferroelectric transition the dielectric function $\varepsilon(\omega)$ and the dielectric modulus $\beta(\omega)$ - the latter one was calculated from the measured dielectric function according to $\beta(\omega)=(\varepsilon(\omega))^{-1}$ - behave in the expected manners. The relaxation strength $\Delta \beta_{2}=$ $\beta_{s 1}-\beta_{s 2}$ of $\beta(\omega)$ is nearly temperature independent (see Eq. (40)) whereas the relaxation strength $\Delta \varepsilon_{2}=\varepsilon_{s 2}-\varepsilon_{s 1}=\bar{\chi}_{s 2}$ of $\varepsilon(\omega)$ strongly increases near $T_{c}$ (see Equation (26)). Figure 4 shows that this behaviour holds at least up to temperatures of about $15 \mathrm{~K}$ below the transition temperature, thus confirming the results of Figure 1 .

The relaxation frequency $\tau_{\varepsilon 2}^{-1}$ depends in a more complicated manner on the temperature. On approaching $T_{c}$ from the lower paraelectric phase this quantity increases reaching a maximum value at about $-22^{\circ} \mathrm{C}$ and then decreases. This behaviour should be compared to that given in Figure 2. In contrast to the assumptions introduced in Fig. 2 the parameter $p$ generally depends exponentially on the temperature according to a thermally activated process. In the case of Rochelle salt $\tau_{d} \gg \tau_{n}$ holds and thus the activation energy of $p$ is nearly equal to that describing the motions of the defects. Consequently it is expected that far away from $T_{c}$ the temperature dependence of $\tau_{\varepsilon 2}^{-1}$ is given by that of $p$, which according to Eq. (28) increases with increasing temperature, whereas close to $T_{c}$ the influence of the phase transition on the dynamical behaviour becomes prominent. It should be noted that because of the same reason the critical relaxation frequency $\tau_{\varepsilon}^{-1}$ of an undisturbed order-disorder ferroelectric shows a similar behaviour near $T_{c}$ [22]. According to 

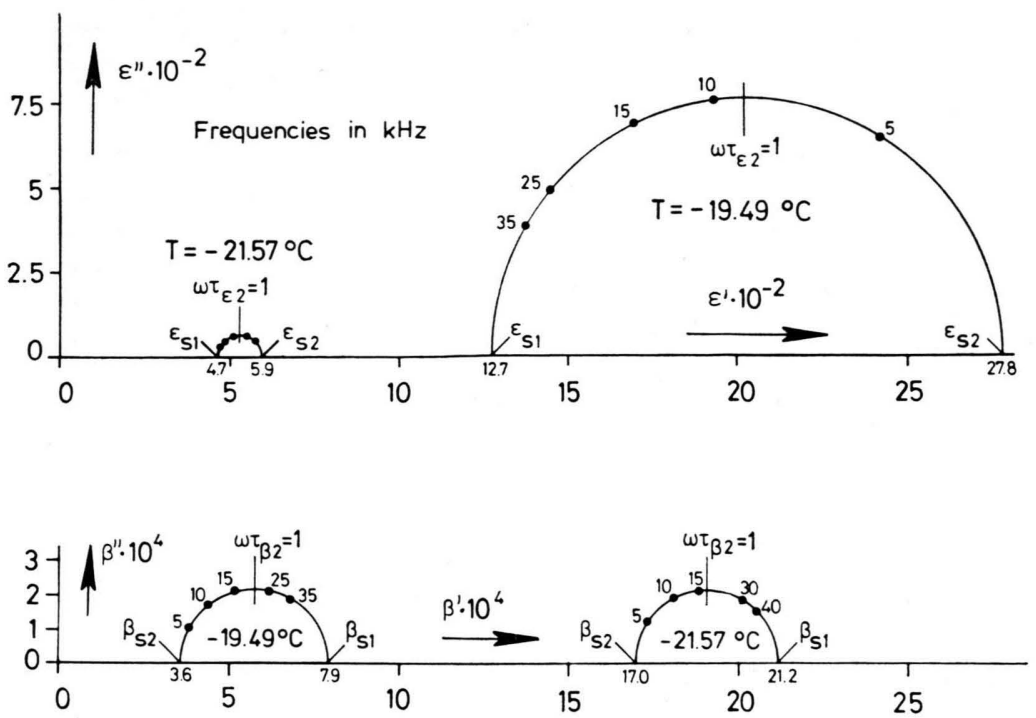

Fig. 3. Temperature and frequency dependences of the complex dielectric constant $\varepsilon(\omega)=\varepsilon^{\prime}(\omega)-i \varepsilon^{\prime \prime}(\omega)$ and the dielectric modulus $\beta(\omega)=\beta^{\prime}(\omega)+i \beta^{\prime \prime}(\omega)$ in the lower paraelectric phase near the ferroelectric transition as observed by Klein [29].
Eq. (43) $\tau_{\beta 2}=\tau_{d}$ should follow a thermally activated process which fits well to the results presented in Figure 5. It is a special feature of the very fast after effect that it cannot be detected in the ferroelectric phase because its interaction with the domains. The results obtained in the upper paraelectric phase fit well to the line extrapolated from the lower paraelectric phase (Fig. 5; it should be noted that it is difficult to realize the condition of a free crystal in this temperature range because $\tau_{\beta 2}$ is considerably smaller than in the lower paraelectric phase; therefore there exist only few measurements in the upper paraelectric phase).

According to Fig. 5 and the data known for the critical dielectric dynamics [3-5] the parameter $p^{\prime}=\tau_{\beta 1} / \tau_{\beta 2}$ is of the order of magnitude $10^{-5}-10^{-8}$ in the investigated temperature range. Because of Fig. 2 and Eq. (28) this result implies that the influence of the defect relaxation on the high frequency critical dynamics is so small that it cannot be resolved experimentally. On the other hand, although the influence of the critical dielectric dynamics on the dynamics of the defects is small it can be detected close to $T_{c}$ (Figure 4). Of course, a similar statement does not hold for the static material quantities, i.e. near $T_{c}$ the relaxation strength $\Delta \beta_{2}$ is of the order of magnitude of the static modulus $\beta_{\delta 1}$. According to Fig. 4 the crystal does not reach the phase transition temperature $T_{c}$. This effect can be attributed to an influence of the other after effects. The phase transition actually appears at a temperature for which the static dielectric modulus vanishes, where static means that all internal transformations are in equilibrium which couple linearly to the order parameter of the perfect crystal. In this sense it may be stated that in Rochelle salt certain defects exist with a characteristic time scale of about $100 \mathrm{~s}$ which contribute linearly to the polarization and which very close to $T_{c}$ drive the phase transition. Obviously this behaviour corresponds to an extreme narrow central peak in the fluctuation spectrum of the polarization.

The following special properties could be proved for the very fast after effect in Rochelle salt (similar

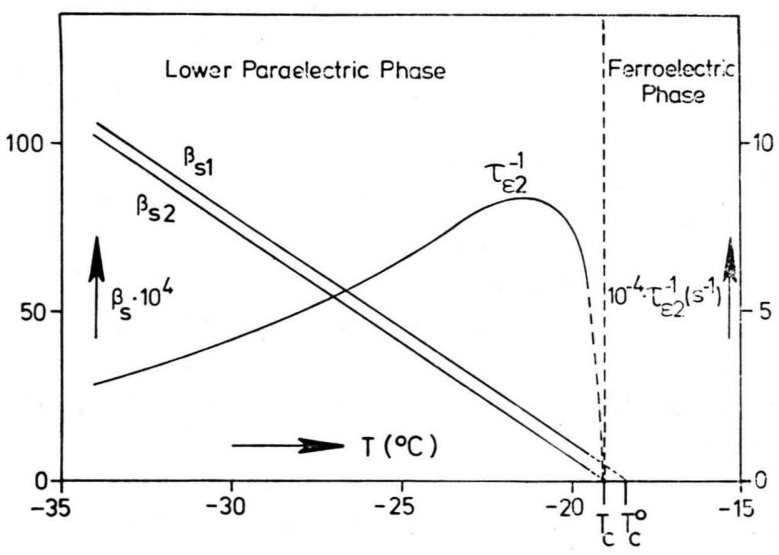

Fig. 4. Schematic representation of the temperature dependences of $\beta_{\mathrm{s} 1}, \beta_{\mathrm{s} 2}$ and $\tau_{\varepsilon 2}^{-1}$ in the lower paraelectric phase as extracted from plots according to Figure 3 . 


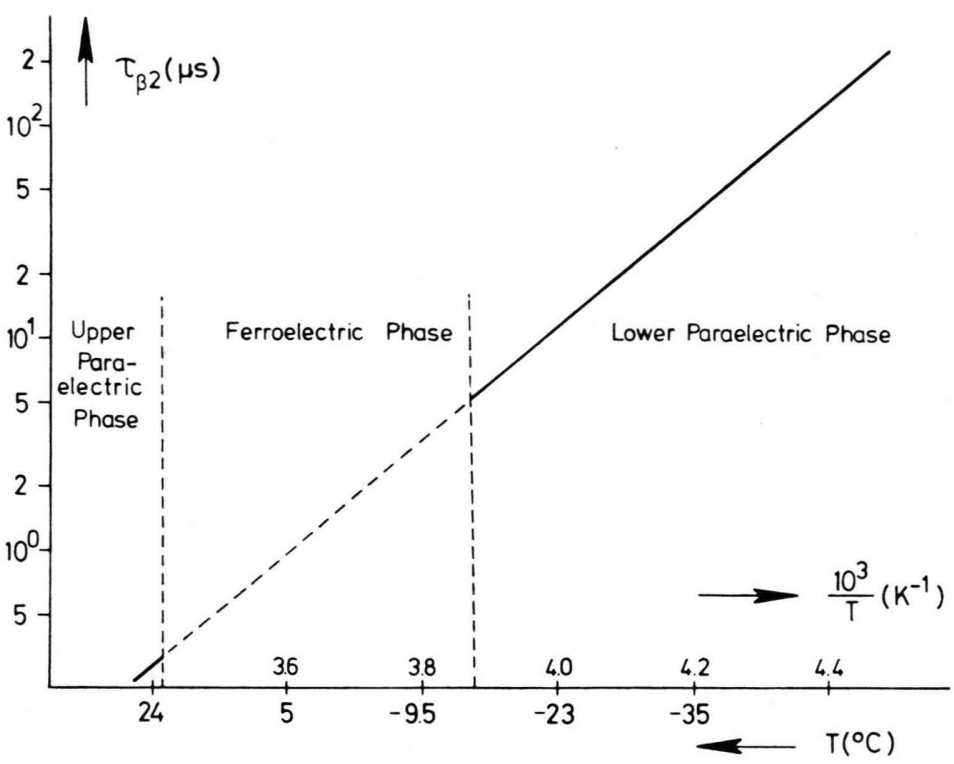

Fig. 5. Arrhenius plot of the relaxation time $\tau_{\beta 2}$ according to $[7,8]$. The slope of the line corresponds to an activation energy of $0.5 \mathrm{eV}$ per particle. statements generally do not hold for the other after effects). Extensive experimental studies [7] showed that the relaxation strength $\Delta \beta_{2}$ of this after effect is strongly influenced by the water content of the Rochelle salt crystal, which can be reversibly regulated by storing the crystal for a sufficiently long time in air of a definite humidity. The authors of this work also succeeded in relating quantitatively the observed behaviour of $\Delta \beta_{2}$ and the equivalent shifts of the transition temperatures to a change of concentration of water vacancies in the lattice. Thus, one of the basic assumptions of the HV model, i.e. the dependence of the central peak behaviour on the concentration of the defects, has been extensively investigated and verified for the very fast after effect in Rochelle salt. Furthermore it has been shown [7, 8] that this after effect cannot be detected in clamped crystals. From thermodynamics it then can be concluded that an elastic relaxation which via the piezoelectric effect couples linearly to the polarization must be regarded as the cause for the observed very fast dielectric relaxation. From the dielectric data the strength of the elastic relaxation could be estimated to be of the order of magnitude of some per cent. A dynamical exchange between crystal water molecules on certain lattice sites and on interstitial sites is assumed to be the most likely molecular mechanism [7]. The observed activation energy of $0.5 \mathrm{eV}$ is ascribed to the process of forming a water vacancy on a lattice site and an interstitial water molecule.

\section{Conclusions}

It has been shown that the $\mathrm{HV}$ model and the LK theory are equivalent methods for describing the interaction of the strongly temperature dependent relaxational polarization mode with relaxational defect modes near the ferroelectric transitions of Rochelle salt crystals. As is well known $[15,16,23,24]$ the LK approach can be extended also to the case that oscillatory modes must be taken into account. This situation obviously corresponds to the original $\mathrm{HV}$ model. Then on the left hand side of Eqs. (34)-(36) besides the relaxational terms also oscillatory terms must be taken into consideration. Also in this case it may be assumed that none of the coefficients in this representation depends in an anomaleous manner on the temperature. Rather the behaviour of the determinants which have been discussed above give rise to the anomaleous static and dynamic behaviour of the material quantities. A difficulty arises now from the fact that it is generally not possible to introduce normalized macroscopic polarization variables, i.e. generally there are mixed linear terms between these internal variables of state. In particular generally it cannot be stated that the macroscopic behaviour of the relaxing defect cell model is given by a linear superposition of a motion of the damped harmonic oscillatory type and a relaxational motion. However, since the variables of state appearing in Eqs. (34)-(36) nearly coincide with 
the variables describing the motions as a function of the local forces it may be reasonably assumed that $P_{\beta 1}$ is given by a damped harmonic oscillatory motion, that $P_{\beta 2}$ is given by a relaxational motion and that both variables or decoupled. One now may transform to relations of the form of Eqs. (30)-(32) - which necessarily contain mixed terms between the variables $P_{\varepsilon 1}$ and $P_{\varepsilon 2}-$ and then discuss the central peak behaviour [16]. Obviously this approach is quite analogous to the original $\mathrm{HV}$ model which starts from a description of the motions

[1] B. I. Halperin and C. M. Varma, Phys. Rev. B 14, 4030 (1976).

[2] J. B. Hastings, S. M. Shapiro, and B. C. Fraser, Phys. Rev. Lett. 40, 237 (1978).

[3] H. E. Müser and J. Pottharst, phys. stat. sol. 24, 109 (1967).

[4] F. Sandy and R. V. Jones, Phys. Rev. 168, 481 (1968).

[5] E. Sailer and H. G. Unruh, Sol. State Com. 16, 615 (1975).

[6] H. G. Unruh, Proc. Europ. Meet. Ferroelectricity, Wissenschaftliche Verlagsgesellschaft, Stuttgart 1970, pp. 167-180.

[7] H. G. Unruh and E. Sailer, Z. Phys. 224, 45 (1969).

[8] G. Klein and G. Luther, Z. Naturforsch. 25a, 1159 (1970).

[9] H. G. Unruh and H. E. Müser, Z. angew. Phys. 14, 121 (1962).

[10] H. G. Unruh, Z. angew. Phys. 16, 315 (1963).

[11] H. E. Müser, Z. Phys. 184, 105 (1965).

[12] H. G. Unruh, Phys. Kondens. Materie 4, 275 (1965).

[13] H. E. Müser and H. Schmitt, J. Phys. Paris 33, C 2, 103 (1972).

[14] H. E. Müser and H. G. Unruh, Z. Naturforsch. 21 a, 783 (1966). of the order parameter and the defects as a function of the corresponding local forces and then derives relations for the macroscopic susceptibility.

\section{Acknowledgement}

The author is indebted to Professors H. Thomas and K. A. Müller for encouraging comments and to the Deutsche Forschungsgemeinschaft for supporting this work within the frame of the Sonderforschungsbereich Ferroelektrika.

[15] H. E. Müser and J. Petersson, Fortschr. Phys. 19, 559 (1971).

[16] J. Petersson, Ferroelectrics 13, 565 (1976).

[17] L. D. Landau and I. M. Khalatnikov, Dokl. Akad. Nauk. SSSR 96, 469 (1954).

[18] R. L. Reese, I. J. Fritz, and H. Z. Cummins, Phys. Rev. B 7, 4165 (1973).

[19] M. Brody and H. Z. Cummins, Phys. Rev. B 9, 179 (1974).

[20] N. Lagakos and H. Z. Cummins, Phys. Rev. B 10, 1063 (1974).

[21] G. Luther, phys. stat. sol. (a) 20, 227 (1973).

[22] J. Grossmann, D. Müller, J. Petersson, and E. Schneider, Z. Naturforsch. 31 a, 1089 (1976).

[23] J. Meixner and H. Reik, Encyclopedia of Physics, Vol. III/2, Springer-Verlag, Berlin 1959, pp. 413-523.

[24] J. Petersson, Ferroelectrics 4, 221 (1972).

[25] R. H. Lyddane, R. G. Sachs, and E. Teller, Phys. Rev. 59, 673 (1941).

[26] J. Petersson, phys. stat. sol. (b) 53, K 79 (1972).

[27] J. Meixner, Z. Naturforsch. 9a, 654 (1954).

[28] A. S. Barker, Phys. Rev. B 12, 4071 (1975).

[29] G. Klein, Diploma Thesis, University of Saarbrücken, 1969. 